\title{
Food entrapped in papilla of Vater: uncommon cause of vomiting
}

\author{
D Falchetti, P Pedersini, W Rigamonti, P Salucci, G Caccia
}

\begin{abstract}
Case report-A 20 month old girl was admitted for intractable vomiting over several days, with no other symptoms. Family and personal history were not contributive. Clinical and neurological examination, and routine blood tests and investigations (plain abdominal $x$ ray, upper gastrointestinal tract contrast study, abdominal ultrasonography) were normal. The upper gastrointestinal endoscopy showed a mild antral gastritis and the second portion of duodenum was occupied by a tough, fibrous mass partially embedded into the papilla of Vater. The foreign body was removed and proved to be vegetable fibre (pineapple). Symptoms subsided immediately and the child was discharged with gastroprotective therapy. After two months, on endoscopic examination, the signs of gastropathy had cleared; the papilla of Vater was undamaged, but unchomped food debris was again found in the duodenum.

Discussion-There are sporadic reports of foreign bodies retained into the papilla of Vater, all of them in adults. This child, though her papilla was tiny, had no jaundice or pancreatitis, unlike most of the reported cases. This is the first report of this finding in a child. The cause of the vomiting was not shown on abdominal ultrasonography or contrast study. It should be added to the list of unusual causes of vomiting.
\end{abstract}

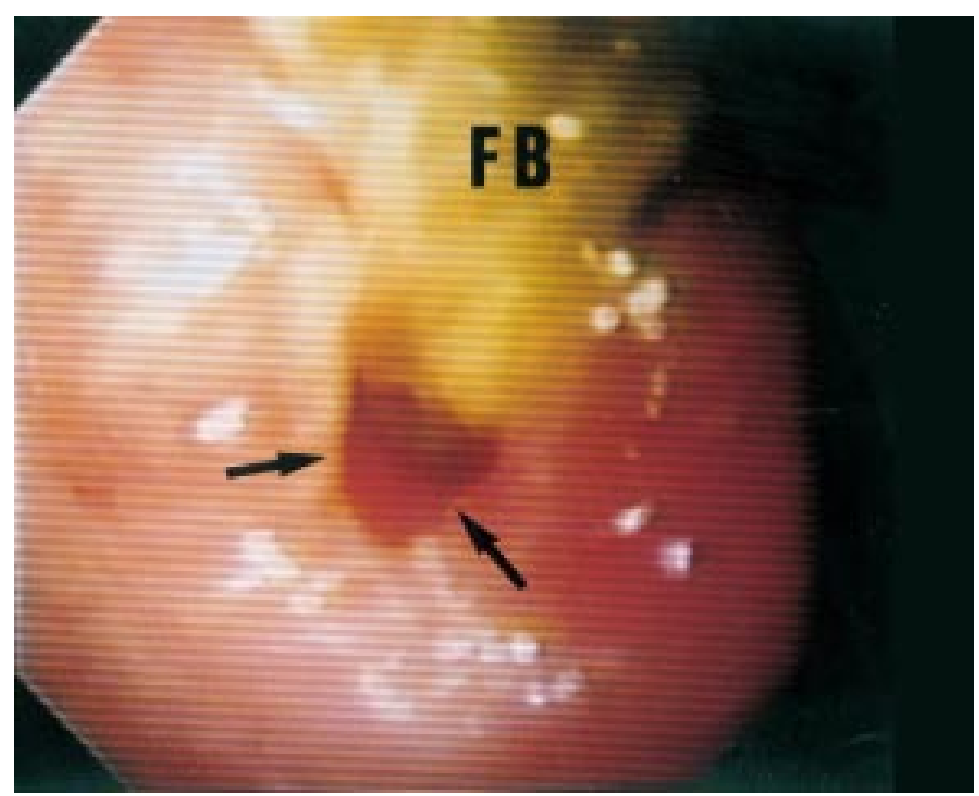

Figure 1 The foreign body (FB) was pivoting, partially embedded in the papilla of Vater (arrows).
(Arch Dis Child 2000;82:488-489)

Keywords: vomiting; children; papilla of Vater; endoscopy

Uncomplicated vomiting is commonplace in the first years of life, and therefore does not usually alarm parents or paediatricians. In most cases the cause of vomiting is easy to identify. ${ }^{1}$ Nevertheless, the paediatric disorders associated with vomiting are so many that the initial investigations may be insufficient to recognise the precise cause, especially if it is uncommon. We report the case of a 20 month old child with a very unusual reason for vomiting.

\section{Case report}

A 19 month old girl was admitted to a second level paediatric department for persistent vomiting for many days, without no other symptoms. Vomiting was mostly after meals without warning; it was never bile stained or associated with severe abdominal pain. Family and remote personal histories were not contributive.

Clinical examination, routine blood tests (including bilirubin and amylase), abdominal ultrasonography, plain abdomen $x$ ray, and upper gastrointestinal tract contrast study were negative. After two weeks, the child was referred to our department for upper gastrointestinal endoscopy.

The investigation showed a normal appearance of the digestive tract until the first portion of duodenum, apart from a slight superficial antral gastropathy. The second portion of duodenum was occupied by a large, frayed, fibrous yellowish foreign body. It was pivoting, partially embedded in the papilla of Vater. The foreign body was removed and proved to be a piece of pineapple which the child had grabbed from her mother's hand and swallowed several days previously (fig 1).

All symptoms subsequently subsided. She was discharged with mild gastroprotective therapy for a month and she remained asymptomatic. After two months, endoscopic monitoring of her erosive antritis showed complete mucosal recovery and a normal appearing papilla. Curiously, after a six hour fast, macroscopic debris of food was found again in the stomach and first portion of the duodenum.

\section{Discussion}

Reports of a swallowed foreign body embedded in the papilla of Vater are exceedingly rare and all are in adults, ${ }^{2-6}$ apart from one case of a child who had previously undergone an endoscopic sphincterotomy. ${ }^{7}$ Jaundice is usually the main 
symptom in this condition, and vomiting is seldom described.

In the case presented, the impacted foreign body did not cause biliopancreatic flow obstruction, despite the tiny dimensions of the papilla. The only effect was increasing vomiting, probably on a reflex basis. Endoscopy is necessary to show this lesion.

1 Walker WA, Durie PR, Hamilton JR, Walker-Smith JA, Watkins JB. Pediatric gastrointestinal disease, Vol. 1. St Louis: Mosby, 1996:198-9.

2 Sferco A, Cantor D, Casal M, Machado E. Foreign body
Odditis. Acta Gastroenterol Latinoam 1970;2:25-7.

3 Rao BK, Lieberman LM. Intermittent common bile duct obstruction at ampulla of Vater due to chicken bone impaction: diagnosis by hepatobiliary imaging. Clin Nucl Med 1981;6:59-61.

4 Zonnebelt S. Wolberg W. Ascending cholangitis secondary to chicken bone lodged at the ampulla of Vater. Am F Surg 1981;141:610-11.

5 Danzi JT. Two cases of acute pancreatitis due to foreign body. Gastrointest Endosc 1986;32:360-1.

6 Meltzer SJ, Goldberg MD. Recurrent pancreatitis caused by vegetable matter obstruction. Am f Gastroenterol 1986;818: 1091-2.

7 Sakai T, Kihara M, Wagayama H, Shimizu A, Kunlyoshi M, Tameda Y. A case of recurrent choledocholithiasis due to foreign bodies (fish bones) after endoscopic sphincterotomy. Nippon Shokakibyo Gakkai Zasshi 1997;94:143-7.

\section{STAMPS IN PAEDIATRICS}

\section{UNICEF}

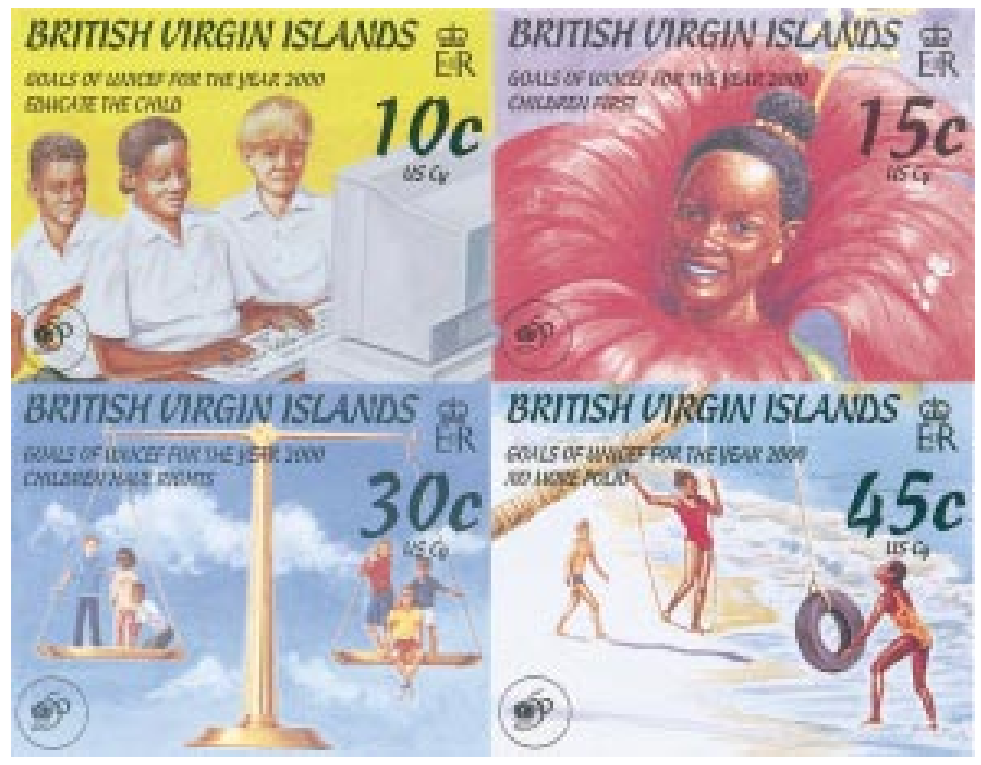

The goals of UNICEF for the year 2000 are illustrated on the set of four stamps from the British Virgin Islands issued in 1996 to mark the 50th anniversary of the organisation. The themes depicted are "Educate the child", "Children first", "Children have rights", and "No more polio". The UNICEF logo incorporating the number 50 is on each of the stamps.

M K DAVIES A J MAYNE 